The Astrophysical Journal, 492:L173-L176, 1998 January 10

(C) 1998. The American Astronomical Society. All rights reserved. Printed in U.S.A.

\title{
2.12 MICRON MOLECULAR HYDROGEN EMISSION FROM CIRCUMSTELLAR DISKS EMBEDDED IN THE ORION NEBULA
}

\author{
HuA CHEN \\ Steward Observatory, University of Arizona, 933 North Cherry Avenue, Tucson, AZ 85721; hchen@as.arizona.edu \\ JOHN BALLY \\ Department of Astrophysical, Planetary, and Atmospheric Sciences, Center for Astrophysics and Space Astronomy, University of Colorado, Boulder, CO \\ 80309-089; bally@ nebula.colorado.edu \\ C. Robert O'Dell \\ Department of Physics and Astronomy, Rice University, Houston, TX 77251; cro@ rice.edu \\ Mark J. McCaughrean \\ Max-Planck-Institut für Radioastronomie, Auf dem Hugel 69, D53121 Bonn, Germany; mjm@mpifr-bonn.mpg.de \\ AND \\ Rodger L. Thompson, Marcia Rieke, Glenn Schneider, and Erick T. Young \\ Steward Observatory, University of Arizona, 933 North Cherry Avenue, Tucson, AZ 85721; thompson@as.arizona.edu, mrieke@as.arizona.edu, \\ gschneider@as.arizona.edu, eyoung@as.arizona.edu \\ Received 1997 July 29; accepted 1997 October 30; published 1997 December 30
}

\begin{abstract}
We present narrowband images of two externally illuminated circumstellar disks embedded in the Orion Nebula. The images were taken with camera 2 of the Near-Infrared Camera and Multiobject Spectrometer (NICMOS) as part of the Hubble Space Telescope Early Release Observations program. Molecular hydrogen emission is confined to the silhouettes embedded in the objects HST 10 (182-413) and HST 17 (183-419). This emission appears to trace the surfaces of nearly edge-on circumstellar disks and is likely to be excited by the intense far-ultraviolet (FUV) radiation field produced by nearby $\mathrm{O}$ stars. The presence of this emission confirms that FUV radiation penetrates to the disk surface. The absence of $\mathrm{H}_{2}$ emission in the region between the ionization front and the disk surface provides evidence that this medium is predominantly atomic. Our observations constitute the first demonstration that the dense circumstellar disks embedded in the proplyds are predominately molecular. While the central star is marginally detected at $2.15 \mu \mathrm{m}$ in HST 10, the central star of HST 17 is clearly detected.
\end{abstract}

Subject headings: ISM: clouds — ISM: individual (Orion Nebula) — ISM: molecules — stars: formation

\section{INTRODUCTION}

Located at a distance of about $430 \mathrm{pc}$ (Warren \& Hesser 1977), the Orion Nebula (M42; NGC 1976) is the nearest $\mathrm{H}$ II region and site of ongoing massive star formation. The principal sources of ionizing radiation are the O6p star $\theta^{1} \mathrm{C}$ in the Trapezium cluster and the $\mathrm{O} 9 \mathrm{star} \theta^{2} \mathrm{~A}$ Ori located several arcminutes to the south. In addition to these massive stars, the nebula contains a cluster of over 700 other young stars with a median age of less than $10^{6} \mathrm{yr}$ (Hillenbrand 1997).

O'Dell, Wen, \& Hu (1993, hereafter OWH) detected intense line emission from ionization fronts that wrap around the residual dense gas that surrounds over 50 young stellar objects (YSOs) born from the Orion molecular cloud. They coined the word proplyd (for "protoplanetary disk") to describe these objects as externally illuminated circumstellar material. The refurbishment of the Hubble Space Telescope (HST) led to the detections of previously unseen features and new objects (O’Dell \& Wen 1994; O'Dell \& Wong 1996), resulting in a catalog of 145 proplyds. Seven appear as extremely opaque disklike objects seen in silhouette against the extended background nebula (McCaughrean \& O'Dell 1996).

Many objects surrounded by bright ionization fronts also contain dark silhouettes near their centers. If they are circumstellar disks, as suggested by OWH and McCaughrean \& O'Dell (1996), direct imaging of these proplyds should yield insights into the disk properties. Two silhouettes, HST 10 (182413) and HST 17 (183-419), are located approximately 1' southeast of $\theta^{1} \mathrm{C}$ Orionis and $1^{\prime}$ northwest of $\theta^{2} \mathrm{~A}$ Orionis. The two names for the objects reflect the common names assigned in the discovery paper $(\mathrm{OWH})$ and the coordinate-based designations used in later papers (O'Dell \& Wen 1994). Both were observed by Bally et al. (1997) with the planetary camera (PC) portion of the Wide Field Planetary Camera 2 (WFPC2) in six optical filters, including F547M (continuum), F502N ([O III]), F631N ([O I $]$ ), F656N (H $\alpha)$, F658N ([N II $]$ ), and F673N ([S II]).

The newly commissioned Near-Infrared Camera and Multiobject Spectrometer (NICMOS) camera on HST is particularly suitable for imaging proplyds. It allows us to penetrate deeper into the highly opaque silhouettes and makes it possible to probe proplyds in various infrared emission lines. As part of HST Early Release observations, we observed both HST 10 and HST 17. In this Letter, we report the first detection of molecular hydrogen emission from the surface of the circumstellar disks of these two proplyds. We combine the optical and near-IR HST images and discuss the excitation of $\mathrm{H}_{2}$ in the proplyds.

\section{OBSERVATIONS}

We used the NIC2 camera to image a field containing both HST 10 and HST 17 at a plate scale of 0.076 pixel $^{-1}$ in three narrowband filters, F187N (Paschen- $\alpha$ ), F212N $\left[\mathrm{H}_{2} v=1-0\right.$ $S(1)$ line], and F215N (line-free continuum), as well as a broadband filter F187W for continuum subtraction. Each image consists of three exposures obtained at three positions in a spiral dithering pattern with offsets of 20.5 pixels. Dithering aids in the elimination of cosmic-ray hits and bad pixels. Images were obtained in the MULTIACCUM mode, in which they are read 
nondestructively every $32 \mathrm{~s}$ following several short readouts at the beginning. The total on-source exposure time was 576 $\mathrm{s}$ in the $\mathrm{F} 212 \mathrm{~N}$ and $\mathrm{F} 215 \mathrm{~N}$ filters and $384 \mathrm{~s}$ in the F187N filter.

The raw science images were first processed through IRAF with the STSDAS task CALNICA for dark current subtraction, flat-fielding, and linearity correction. We used on-orbit flat fields for F212N and F215N images. The on-orbit flat is not yet available for F187N and F187W, and we have to use the flat field constructed from the ground thermal vacuum tests. The linearity correction is accomplished by fitting and extrapolating the unsaturated pixels in the multiple readouts. The value of each pixel (in analog-to-digital converter units [ADU] $\mathrm{s}^{-1}$ ) in the output image was determined by the linear regression of the multiple readouts of the same pixel. The background from the warm optics of HST was minimized by our choice of $1 \%$ narrowband filters and was stable during our observations. Therefore, we did not remove the background in the image processing.

We used the STSDAS task CALNICB to register and mosaic the dithered images. The final F187N, F212N, and F215N images are displayed in Figures $1 a-1 c$ (Plate L51). Figure $1 d$ shows the positions of the two proplyds, the field scale and orientation, and the directions to $\theta^{1} \mathrm{C}$ and $\theta^{2} \mathrm{~A}$ Ori, the two main illuminating sources. The relative spatial shifts among the images are less than 0.2 pixel because of the excellent tracking of $H S T$, allowing us to compare the images at subpixel accuracy.

Since the absolute flux calibration is not yet available for the our filters, we rely on differential photometry to calibrate the flux scales. Fortunately, there is one star, PC 228 (not shown in Figs. 1 and 2), in our images that has been observed extensively from the ground at optical and near-infrared wavelengths (Prosser et al. 1994; Hillenbrand 1997; M. J. McCaughrean, unpublished data). By fitting a simple reddened blackbody spectrum to the existing broadband photometry between 0.9 and $3.6 \mu \mathrm{m}$, we were able to interpolate the fluxes (in janskys) through the three NIC2 filters to an uncertainty of less than $15 \%$. Comparing these flux values with the actual measurements (in $\mathrm{ADU} \mathrm{s} \mathrm{s}^{-1}$ ), we obtained flux conversion factors of $5.07 \times 10^{-5}, 4.11 \times 10^{-5}$, and $4.44 \times 10^{-5} \mathrm{Jy}$ $\left(\mathrm{ADU} \mathrm{s}^{-1}\right)^{-1}$ for F187N, F212N, and F215N, respectively. Applying this scale to $\mathrm{F} 215 \mathrm{~N}$ image, we estimate the $5 \sigma$ photometric sensitivity to be $K=17.8$.

\section{RESULTS}

\subsection{Morphology}

We compare the near-infrared NICMOS images with the optical WFPC2 images and discuss the general morphology of the two objects. Figure 2 (Plate L52) displays the composite color images: (a) $\mathrm{Pa} \alpha$ (red)-H $\alpha$ (green)-[O I] (blue); (b) F215N (red)-F547M (green and blue); (c) [N II]-H $\alpha-[\mathrm{O}$ III]; and (d) $\left[\mathrm{H}_{2}\right]-[\mathrm{O} \mathrm{I}]-[\mathrm{S} \mathrm{II}]$. For direct comparison, the WFPC2 images have been rescaled and rotated to match the NICMOS images to subpixel precision. Both NICMOS Pa $\alpha$ and $H_{2}$ images are continuum-subtracted with flux-calibrated F187W and F215N images, respectively. There is no continuum subtraction in the optical line images since our F547M image suggests that the continuum is negligible.

HST 10 consists of a teardrop-shaped ionization front visible in all observed ionized species, in the $\lambda 6300$ line of [O I], in $\mathrm{H} \alpha$, in Paschen- $\alpha$ (Fig. 2a), and to some extent in both the F547M and F215N continuum images (Fig. 2b). The HST 10 ionization front is about 1".0 × 2".6 $(430 \times 1118 \mathrm{AU})$ in extent and elongated along a nearly north-south axis. The brightest part of the ionization front lies at the northern end of the object (see also Fig. 1), which indicates that the dominant source of illumination is $\theta^{1} \mathrm{C}$ Ori. However, the major axis of HST 10 differs from the orientation of a line connecting HST 10 with $\theta^{1} \mathrm{C}$ Ori by about $30^{\circ}$.

In the filters that transmit the light of [O III], [N II], $\mathrm{H} \alpha$, [S II], and $\mathrm{Pa} \alpha$ and in the F547M continuum image, a prominent dark object can be seen in silhouette. The silhouette is elliptical and about $00^{\prime \prime} 10 \times 0.4(40 \times 170 \mathrm{AU})$ in extent, with a major axis oriented orthogonal to the major axis of the ionization front in which it is embedded. This structure may be a nearly edge-on circumstellar disk seen in silhouette against the background nebular light.

HST 17 is a small, nearly circular object with an ionization front about 0"6 (260 AU) in diameter with a 0"10 × 0.30 $(40 \times 120 \mathrm{AU})$ disk seen in silhouette embedded within. In the $\mathrm{H} \alpha$ and $\mathrm{Pa} \alpha$ images, the brightest portion of the ionization front lies to the southeast (see also Fig. 1), which indicates that this objects is illuminated by $\theta^{2}$ A Ori and not by members of the Trapezium.

\section{2. $\mathrm{H}_{2}$ Emission from Disks}

Figure $1 e$ shows an image of the $v=1-0, J=3-1 S(1)$ line of $\mathrm{H}_{2}$ at $\lambda=2.1218 \mu \mathrm{m}$ obtained by subtracting the $\mathrm{F} 215 \mathrm{~N}$ image from the F212N image, The molecular hydrogen emission is entirely confined to the central silhouettes in both HST 10 and HST 17. Unlike [O I] emission, which is produced by both the disk surface and at the ionization front that surrounds these proplyds, $\mathrm{H}_{2}$ emission is produced only in the disks. The emission appears to be extended in HST 10 (FWHM =0"33) and slightly extended in HST 17 (0.22), compared with the point sources (less than 0.2). HST 10 is elongated along the major axis of the silhouette seen in the optical images. The total line luminosity is $2.0 \times 10^{-5} L_{\odot}$ for HST 10 and $8.3 \times 10^{-6} L_{\odot}$ for HST 17 .

\subsection{Continuum Emission}

Both the F547M and F215N continuum images show nearly identical emission from the extended envelopes that surround the central disks (Fig. 2b). This emission is diffuse and extends out to the ionization front, where it reaches its maximum brightness. Since both F547M and F215N were designed to be free of emission lines, their passbands are likely to be dominated by either free-free continuum emitted by a plasma and/or by radiation scattered by dust.

Because the same UV radiation that produces free-free continuum emission should also be responsible for exciting the recombination line emission of atomic hydrogen, we can use our $\mathrm{Pa} \alpha$ line image to estimate the nebular continuum emission. In this calculation, we used the $\mathrm{Pa} \alpha$ recombination coefficient from Osterbrock (1974) and the H I and He II continuous emission coefficients from Ferland (1980), both calculated for $T=$ $10,000 \mathrm{~K}$ and case $\mathrm{B}$ recombination. For a visual extinction of $A_{v}=2.2$ (O'Dell 1997), we calculated the continuum emission within F187N and F215N filters. Convolving the emission with the filter transmission functions, we obtained an F187N-toF215N ratio of 22.3. Subtracting the scaled F187N image from the $\mathrm{F} 215 \mathrm{~N}$ image, we display the residual image in Figure $1 f$. The teardrop-shaped continuum around HST 10 is almost entirely subtracted out. HST 17 is also apparently more compact than it is in the $\mathrm{F} 215 \mathrm{~N}$ image (Fig. 1b).

This suggests that the extended continuum emission is 
mostly the free-free or bound-free nebular light. The compact emission in HST 17 is most likely emission from the stellar photosphere and/or its circumstellar dust. There is also weak residual emission toward HST 10 and its peak coincides with the opaque disk.

\subsection{The Central Stars}

The central stars of HST 10 and HST 17 are not visible in the F547M filter. As discussed in the preceding section, the central star of HST 17 is detected as a prominent continuum feature at $2.15 \mu \mathrm{m}$ (Fig. $1 f$ ). The HST 17 central star has a magnitude of $K=15.9$. Though there is marginal evidence for some continuum emission, no central star is seen in the HST 10 silhouette. This is also illustrated in the color composite image made from the F215N and F547M images (Fig. 2b). While the edge-on disk in HST 10 is still seen in silhouette, the central star in HST 17 overwhelms the appearance of the disk in this object.

The lack of detection of a $2 \mu \mathrm{m}$ star toward the HST 10 disk in our NICMOS image allows us to estimate a minimum extinction through the disk. Assuming that the central star has a magnitude comparable to that of the median unreddened star in the Trapezium cluster, or $M_{K}=12$ (McCaughrean \& Stauffer 1994). Our limiting magnitude of $K \sim 18$ would yield an extinction of $A_{K}>6$ mag or a visual extinction to the central star of at least $60 \mathrm{mag}$. For a normal gas-to-dust ratio, this implies a column density of $N\left(\mathrm{H}_{2}\right)>6 \times 10^{22} \mathrm{~cm}^{-2}$.

\section{DISCUSSION}

\subsection{Ionization Fronts}

The outer parts of HST 10 and 17 represent ionization boundaries, an interpretation demonstrated by the agreement of HST 10 's peak surface brightness with the predictions of photoionization models at the projected distance from $\theta^{1} \mathrm{C}$ Ori (O'Dell $\&$ Wen 1994). Circumstellar disks are embedded in the interior neutral zone and are seen in silhouette in most passbands such as the hydrogen recombination lines, in the continuum, and in all ionized species. However, the disk is bright in the $6300 \AA$ [O I] transition. Comparison of [O I] and [S II] (Fig. 2d) is highly instructive since both lines require roughly $2 \mathrm{eV}$ collisions to excite. In the presence of Lyman continuum radiation, both lines are excited in a thin layer exactly at the hydrogen ionization boundary, since both states would be photoionized in the main body of the ionized gas, and yet they require excitation by the few electron volt electrons found in the fully ionized gas. This is why the outer parts of HST 10 are so similar in $\left[\mathrm{O}_{\mathrm{I}}\right]$ and $[\mathrm{S} \mathrm{II}]$. However, the appearance of the circumstellar disks is quite different in the two ions, with [S II] being absent while [O I] is intense, indicating that sulphur is mostly in its neutral state. The absence of [S II] implies that the density in [O $\mathrm{I}]$ emission must be sufficiently high that recombinations with electrons produced by low-ionization-potential species dominate photoionization.

Since the [O I] transition lies $1.97 \mathrm{eV}$ above the ground state, it requires rather energetic collisions to excite it. If the collision partners are distributed according to a Boltzmann distribution, this requires a gas temperature of at least $3000 \mathrm{~K}$. The [O I] emission at the ionization front is indeed consistent with the model of high-density photodissociation regions (PDRs) proposed by Burton, Hollenbach, \& Tielens (1990). The [O I] emission associated with a silhouette is located at the base of the PDR, where the temperature is much lower, which raises the possibility that the [O I] emission is produced by a nonequilibrium process, an alternative that will be discussed in Bally et al. (1997).

\section{2. $\mathrm{H}_{2}$ Emission Mechanisms}

We first explore internal mechanisms for the excitation of the observed $\mathrm{H}_{2}$ emission that are powered by processes from within the environment of these YSOs. In many T Tauri stars, the $\mathrm{H}_{2} S(1)$ line has been observed in emission (Greene \& Lada 1996). In these systems, the emission is likely to originate either in the inner few astronomical units of an accretion disk, where stellar UV radiation can excite the $\mathrm{H}_{2}$ molecule (Black \& van Dishoeck 1987; Sternberg 1990) or where supersonic shocks heat the gas to temperature in excess of $1000 \mathrm{~K}$ and $\mathrm{H}_{2}$ is collisionally excited. In the former mechanism (UV fluorescence), absorption occurs in the Lyman and Werner bands of $\mathrm{H}_{2}$ below $1100 \AA$ and is followed by a radiative cascade through the various vibrational and rotational states. This mechanism is characterized by high $\left(T_{\mathrm{ex}} \gg 8000 \mathrm{~K}\right)$ vibrational and rotational excitation temperatures. Shock excitation, on the other hand, tends to produce a much lower $\left(T_{\mathrm{ex}} \sim 2000 \mathrm{~K}\right)$ excitation temperature. Thus, these two mechanisms can in principle be distinguished by measurements of the intensities of other transitions. In our case, we can rule out fluorescent excitation by the low-mass stars embedded in each disk. The absence of any sign of the central stars implies high visual and even higher UV extinction to the central stars at UV wavelengths. Thus, UV excitation can produce $\mathrm{H}_{2}$ emission only in the immediate vicinity of the embedded YSO. Our observations show that the $\mathrm{H}_{2}$ emission is extended, and its surface brightness is constant across the observed face of the disk, making it impossible for the illuminating source to be within the disk.

A second possibility is either that the $\mathrm{H}_{2}$ emission is produced in an accretion shock produced by an infalling envelope or that some sort of internal excitation of the disk, such as a spiral density wave, may be producing the excitation. This possibility can be excluded on the grounds of energetics. At the $100 \mathrm{AU}$ outer extent of the HST 10 disk, the gravitational potential of a $1 M_{\odot}$ star would produce a maximum infall velocity of only about $3 \mathrm{~km} \mathrm{~s}^{-1}$. Furthermore, spiral shocks would be restricted to having velocities smaller than this value. Since $\mathrm{H}_{2} v=1-0$ $S(1)$ excitation requires a shock velocity of at least $15 \mathrm{~km}$ $\mathrm{s}^{-1}$ (Chernoff, Hollenbach, \& McKee 1982), the above infall velocity is an order of magnitude too small to collisionally excite $\mathrm{H}_{2}$ into emission in a shock. Furthermore, there is no evidence that the $\mathrm{H}_{2}$ emission is produced by a bipolar outflow from the central YSO. Such an outflow would produce shocks that would extend along the axis of the disk, which is inconsistent with the observed morphology. An outflow might interact with a flared disk and produce shocked $\mathrm{H}_{2}$ emission. Such an emission, however, would be centrally bright because of the $r^{-2}$ divergence of the outflow. This is in disagreement with the observed smooth $\mathrm{H}_{2}$ surface brightness. There are thus no viable internal mechanisms to excite the $\mathrm{H}_{2}$ emission.

The most likely explanation is that the $\mathrm{H}_{2}$ is excited from the outside by the intense UV irradiation of the nearby $\mathrm{O}$ stars. Indeed the observed $\mathrm{H}_{2}$ emission $\left(\sim 10^{-5} L_{\odot}\right)$ matches well with what is predicted by the PDR model (Burton et al. 1990). The fluorescent mechanism produces extended $\mathrm{H}_{2}$ emission from the neutral layer that lies behind the Orion Nebula. The disk emission is seen as an enhanced component superposed on this background. The high angular resolution of our HST obser- 
vations and our data reduction procedures have effectively eliminated this background, which ground-based observations show to be smooth on the angular scales covered by our observations. The NICMOS observations demonstrate that an intense FUV radiation field is incident on the disk surfaces of HST 10 and HST 17. This requires that the total column density between the ionization front and the disk surface be less than about $N(\mathrm{H})=10^{21} \mathrm{~cm}^{-2}$. The absence of extended $\mathrm{H}_{2}$ emission between the disk surface and the ionization indicates that gas in the proplyd envelope is predominantly atomic. Our observations constitute the first demonstration that the dense circumstellar disks embedded in the proplyds are predominantly molecular.
It is our pleasure to acknowledge the NICMOS instrument definition team for their more than one decade of dedication that makes these observations possible. We are grateful to Abi Saha for coordinating the Early Release Observation programs and to Eddie Bergeron and Howard Bushouse for their help in the data reduction. We also like to thank our referee for insightful suggestions that improved this Letter. This work is supported in part by NASA grant NAG5-3042 to the NICMOS instrument definition team. C. R. O. was supported in part by NAG5-1626 to Rice and by the German Humboldt Foundation while a guest of the Max-Planck-Institut für Astronomie in Heidelberg. J. B. was supported in part by NASA grant NAGW 3192 (Long-Term Space Astrophysics).

\section{REFERENCES}

Bally, J., Sutherland, R., Devine, D., \& Johnstone, D. 1997, in preparation Black, J. H., \& van Dishoeck, E. F. 1987, ApJ, 322, 412

Burton, M. G., Hollenbach, D. J., \& Tielens, A. G. G. M. 1990, ApJ, 365, 620

Chernoff, D. F., Hollenbach, D. J., \& McKee, C. F. 1982, ApJ, 259, L97

Ferland, G. J. 1980, PASP, 92, 596

Greene, T. P., \& Lada, C. J. 1996, AJ, 112, 2184

Hillenbrand, L. A. 1997, AJ, 113, 1733

McCaughrean, M. J., \& O’Dell, C. R. 1996, AJ, 111, 1977

McCaughrean, M. J., \& Stauffer, J. R. 1994, AJ, 108, 1382
O’Dell, C. R. 1997, AJ, submitted

O’Dell, C. R., \& Wen, Z. 1994, ApJ, 436, 194

O'Dell, C. R., Wen, Z., \& Hu, X. 1993, ApJ, 410, 69

O'Dell, C. R., \& Wong, S.-K. 1996, AJ, 111, 846

Osterbrock, D. E. 1974, Astrophysics of Gaseous Nebulae (San Francisco: Freeman)

Prosser, C. F., Stauffer, J. R., Hartmann, L., Soderblom, D. R., Jones, B. F., Werner, M. W., \& McCaughrean, M. J. 1994, ApJ, 421, 517

Sternberg, A. 1990, ApJ, 361, 121

Warren, W. H., \& Hesser, J. E. 1977, ApJS, 34, 115 


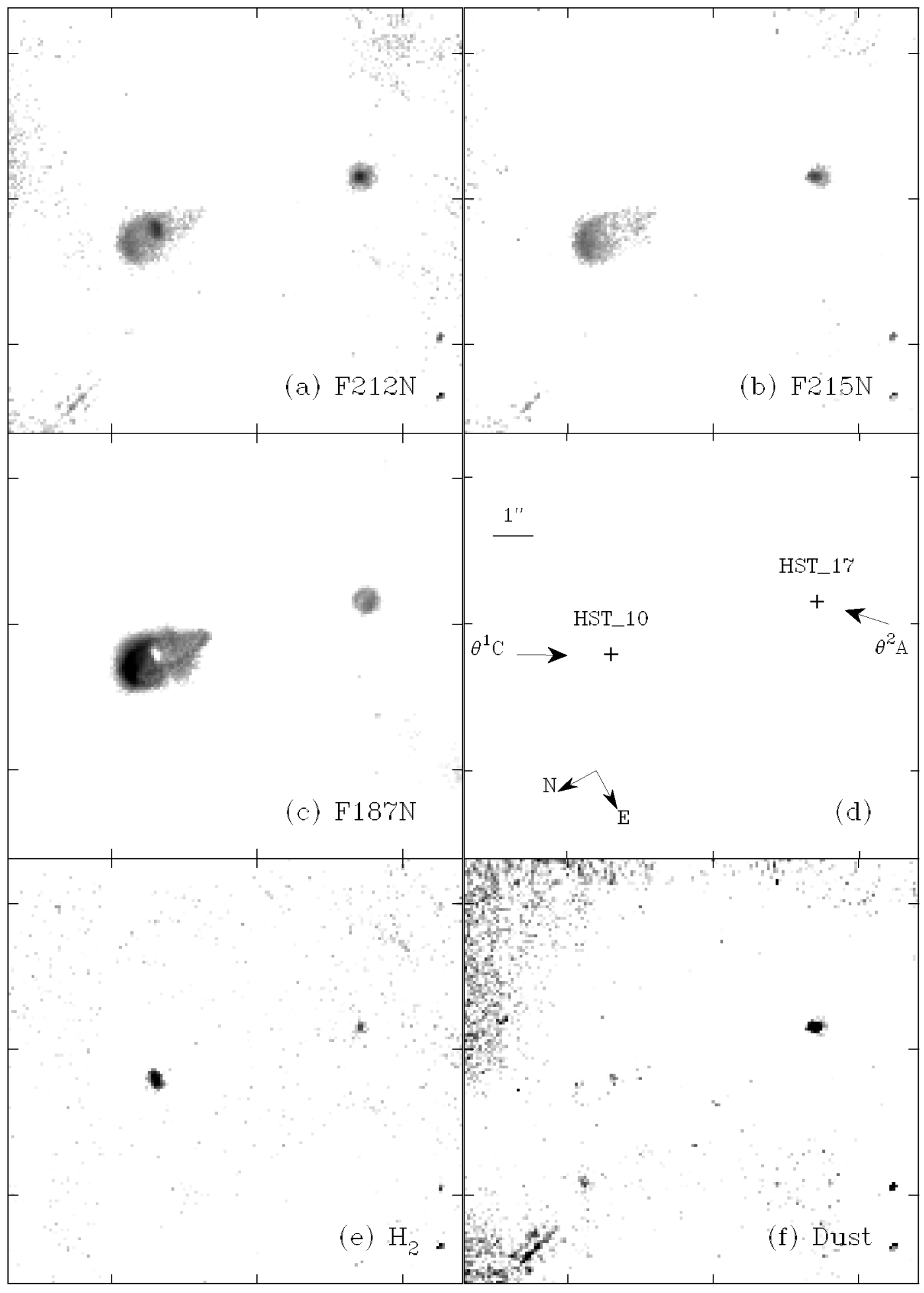

FIG. 1.-HST 10 and HST 17. The images were taken with NICMOS camera 2 at a plate scale of 0 ".076 pixel ${ }^{-1}$. Panels $(a)-(c)$ display three narrowband images; panel $(d)$ shows the positions of the two proplyds, the image scale $\left(1^{\prime \prime}=430 \mathrm{AU}\right)$, and orientation. The two arrows indicate the directions of incoming UV radiation from two nearby massive stars; panel $(e)$ displays the continuum-subtracted $\mathrm{H}_{2}$ emission; panel $(f)$ displays the nebula-subtracted thermal emission $($ see $\S 3.3)$.

CHEN et al. (see 492, L174) 

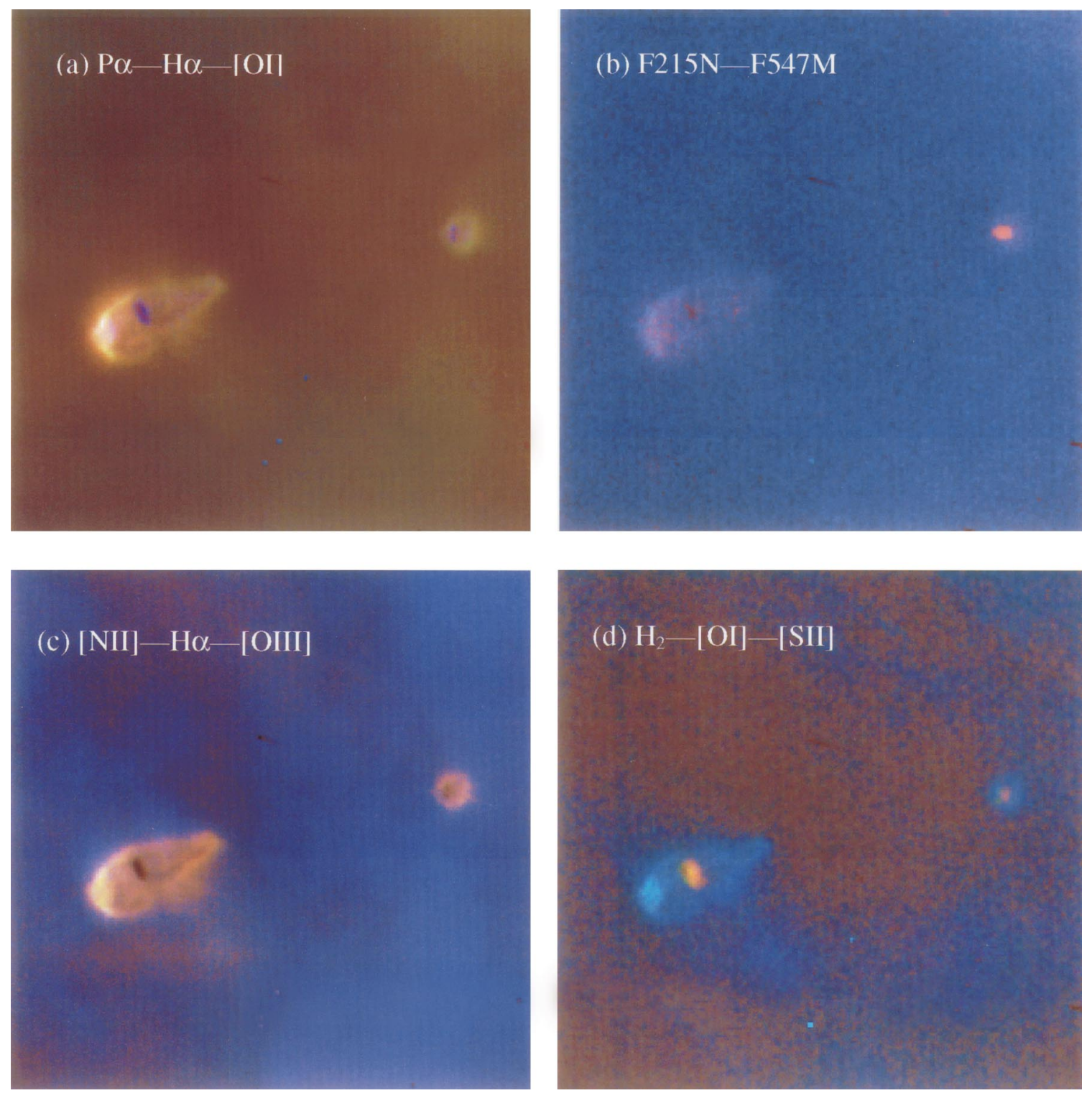

FIG. 2.-Composite color images of HST 10 and HST 17. (a) Pa $\alpha$ (red)-H $\alpha$ (green)-[O I] (blue); (b) F215N (red)-F547M (green and blue); (c) [N II]-H $\alpha-$ [O III]; and $(d)\left[\mathrm{H}_{2}\right]-[\mathrm{O} \mathrm{I}]-[\mathrm{S}$ II]. For direct comparison, the WFPC2 images have been rescaled and rotated to match the NICMOS images to subpixel precision.

ChEn et al. (see 492, L174) 